\author{
M. IZQUIERDO* \\ $X$. AGUADO* \\ J. L. GONZÁLEZ* \\ J. L. LÓPEZ* \\ T. RIBAS** \\ F. LINARES** \\ L. VILA*** \\ J. A. VOCES*** \\ A. I. ÁLVAREZ*** \\ J. G. PRIETO****
}

* Laboratorio de Biomecánica del Instituto Nacional de Educación Física de León.

** Servicio de Anatomía Patológica. Complejo Hospitalario de León.

*** Departamento de Fisiología, Farmacología y Toxicología. Universidad de León.

\title{
Características musculares y producción de fuerza máxima/explosiva durante acciones isométricas/dinámicas de los músculos extensores de los miembros inferiores en jóvenes no entrenados Muscle characteristics and maximal/explosive force production during isometric/dynamic actions of leg extensor muscles in untrained young men
}

\section{Resumen}

Participaron en el estudio, de forma voluntaria cinco sujetos de sexo masculino, estudiantes de educación fisica (22-26 años). El objetivo fue observar las relaciones entre el test de salto horizontal con los tests de salto vertical con contramovimiento y con flexión previa, tests de fuerza isométrica máxima y la distribución de los tipos de fibras musculares.

Los resultados en la distancia y altura de vuelo conseguidas en los tests de salto horizontal, salto desde flexión previa sin contramovimiento $y$ salto con contramovimiento fueron respectivamente $2,53 m \pm 0,17,0,32 m \pm 0,05$ y $0,40 m \pm 0,05$. No se encontraron correlaciones significativas entre las fibras tipo I, tipo IIA y tipo IIB $(48 \% \pm 1,34 \% \pm 3,16 \% \pm 4$, respectivamente) y los tests realizados. Tampoco se encontraron correlaciones significativas entre la máxima fuerza, la pendiente de fuerza isométrica y entre la distancia y alturas de salto. Por otro lado, tampoco se encontraron correlaciones entre la fuerza y la potencia en el salto horizontal $(2,53 \mathrm{BW}$ $\pm 0,17$ y $63,4 \mathrm{~W} / \mathrm{kg} \pm 5,4$, respectivamente) y la distancia lograda.

El test de fuerza isométrica máxima se realizó con dos diferentes ángulos de flexión de rodillas: $90^{\circ}$ y $120^{\circ}$. Tanto el pico de máxima fuerza isométrica como la máxima pendiente de desarrollo de la fuerza fueron superiores al realizar el test con una flexión de rodillas de $120^{\circ}(2268 \mathrm{~N} \pm 576$ y $8962 \mathrm{~N} / \mathrm{s} \pm 1945$ frente a $1538 \mathrm{~N} \pm$ 300 y $7398 \mathrm{~N} / \mathrm{s} \pm 1557$ con $90^{\circ}$ de flexión). Por último, no se encontraron relaciones significativas entre la fuerza isométrica máxima y la fuerza explosiva en acciones dinámicas.

Palabras clave: Fuerza explosiva. Fuerza isométrica. Salto horizontal a pies juntos. Test de salto vertical.

\section{Summary}

A group of five physical education students men (22-26 years) volunteered as subjects for examination the relationship between standing long-jump, countermovement jump, squat jump, maximal and explosive isometric force production and muscle fiber distribution.

Height and distance attained in the standing long.jump test, squat jump and countermovement jump was $2.50 \pm 0.17 \mathrm{~m}, 0.32$ $\pm 0.05 \mathrm{~m}$ and $0.40 \pm 0.05 \mathrm{~m}$. No significant correlations were found between type I, type IIA and type IIB fibers and the test results $(48 \pm 1 \%, 34 \pm 3 \%, 16 \pm 4 \%$, respectively). Likewise, no significant correlations were found between maximum force, the isometric force slope, and jump lengths and heights. No correlation was found between strength and power $(2.53 \pm 0.17 \mathrm{BW}$ and 63.4 $\pm 5.4 \mathrm{~W} / \mathrm{kg}$, respectively) and the distance achieved in the long standing jump.

The maximum isometric strength test was carried out using two different knee flexion angles: $90^{\circ}$ and $120^{\circ}$. Peak isometric force and the maximum force slope were greater when the test was carried out with $120^{\circ}$ knee flexion $(2268 \pm 576 \mathrm{~N}$ and $8962 \pm 1945 \mathrm{~N} / \mathrm{s}$ versus $1538 \pm 300 \mathrm{~N}$ and $7398 \pm 1557 \mathrm{~N} / \mathrm{s}$ with $90^{\circ}$ flexion). Finally, no significant relation were found between peak isometric force and explosive force in dynamic actions.

Key words: Explosive force. Isometric force. Standing long jump. Vertical jump.

Correspondencia:

M. IZQUIERIDO. Instituto Nacional de Educación Física de León. Laboratorio de Biomecánica. Campus de Vegazana, s/n. 24071 León. 


\section{Introducción}

Ia capacidad del sistema neuromuscular para desarrollar rápidamente un nivel de fuerza tiene especial relevancia en actividades no sólo deportivas, síno también, de la vida cotidiana ${ }^{1}$. En éstas, el tiempo que se tarda en conseguir un determinado nivel de fuerza será tan importante como la máxima fuerza que un individuo pueda desarrollar².

Por otro lado, la manifestación explosiva de la fuerza es una de las cualidades más valorada por los investigadores al diseñar baterías de pruebas físicas que discriminen individuos con diferentes aptitudes. Así se utiliza junto con otras pruebas, en los accesos a Inefs, a cuerpos de seguridad y también en el control de atletas de diferentes especialidades ${ }^{3}$.

El Squat Jump (SJ), el Countermovement Jump $(\mathrm{CMJ})^{4,5}$ y el test de salto horizontal a pies juntos desde parado (SHP) ${ }^{6}$, son los más utilizados cuando se evalúa la capacidad de los músculos extensores de los miembros inferiores para generar potencia mecánica durante un corto período de tiempo ${ }^{7}$.

Las fibras musculares de contracción rápida (tipo II) son capaces de desarrollar tensión muscular más rápidamente que las de contracción lenta (tipo I). Así, no es extraño encontrar relaciones significativas entre el porcentaje de fibras tipo II y la fuerza producida en acciones que requieren altas velocidades de salida o de impacto en los cuerpos como, por ejemplo, las carreras de velocidad, los lanzamientos o los saltos ${ }^{8-10}$.

El movimiento humano combina acciones musculares de típo isométrico y dinámico. Sin embargo, es frecuente que en condiciones de experimentación se explore aisladamente un solo tipo de contracción $\mathrm{n}^{2}, 11,12$.

Tradicionalmente los investigadores, en experiencias con sujetos jóvenes, han extrapolado los resultados de la fuerza explosiva obtenidos en tests isométricos a acciones dinámicas. En las contracciones isométricas la pendiente máxima de fuerza (PMF) es la variable que mejor se relaciona con la fuerza explosiva. Sin embargo, mientras que para unos autores la PMF se correlaciona significativamente con movimientos explosivos en contracciones dinámicas ${ }^{13-16}$, otros muestran que no sucede, por ejemplo, respecto a las carreras de velocidad ${ }^{17,18}$ o a la capacidad de salto $^{19}$.

Sin embargo, en el SHP, no se conoce con certeza la relación que pueda existir entre la distancia del salto, las características musculares del sujeto y la manifestación explosiva de la fuerza (ya sea estimada mediante acciones de tipo isométrico o dinámico).

El objetivo de este trabajo fue analizar la relación entre los índices de fuerza explosiva durante los saltos y la producción bilateral de fuerza isométrica en los músculos extensores de los miembros inferiores. En segundo lugar, identificar la relación entre la distribución de los tipos de fibras musculares I, IIA y IIB del vasto lateral y los diferentes tests de salto.

\section{Material y método}

\section{Sujetos}

Participaron en el estudio, de forma voluntaria, cinco sujetos de sexo masculino $(X=23$ años \pm 1$)$. Todos ellos eran estudiantes de educación física, con una práctica regular de actividad física de tres veces por semana. Para mantenerse en forma, realizaban actividades como ir en bicicleta o correr. Ninguno de ellos había entrenado nunca regularmente la fuerza ni había participado en deportes de competición. La altura, peso corporal y porcentaje de tejido graso fueron $1,80 \mathrm{~m} \pm 9,78 \mathrm{Kg} \pm 10$ y $13,5 \% \pm 2$, respectivamente. Todos los sujetos fueron informados de los procedimientos y posibles repercusiones del trabajo, pidiéndoles voluntariamente por escrito su conformidad para participar en el mismo. El trabajo obtuvo la conformidad de las comisiones de étíca de la Universidad de León y del Complejo Hospitalario de León.

\section{Mediciones de fuerza y tests de salto}

Se familiarizó a los sujetos con los protocolos utilizados en las mediciones de fuerza. Para ello se realizaron, una semana antes varios intentos submáximos y máximos. Además, el día de las mediciones, que fue el día anterior a las biopsias, se permitieron varias repeticiones de calentamiento previamente a la ejecución del test.

La fuerza explosiva dinámica fue estimada utilizando una plataforma de fuerzas extensiométrica (Dinascan $600 \mathrm{M}, \mathrm{IBV}$ ). Para ello se realizó un SJ desde la posición de $90^{\circ}$ de flexión de rodillas, un $\mathrm{CMJ}$ dejando libre la flexión previa al salto y un SHP.

El SJ se realizó con las manos sobre las caderas durante todo el movimiento, para asegurarse de que fuera ejecutado sin la ayuda de los brazos. Por otro lado, no se permitió la ayuda del tronco en el salto, al dar instrucciones para que éste se mantuviera vertical durante la impulsión. También se dieron instrucciones para que los sujetos aterrizaran en una posición igual a la adoptada en el instante del despegue. Los intentos en los que previamente a la contracción concéntrica aparecía una fase de contramovimiento superior al $5 \%$ de la fuerza máxima fueron eliminados. La altura de vuelo y la potencia mecánica fueron calculadas a partir del tiempo de vuelo.

El SHP fue realizado siguiendo el protocolo descrito en la Batería Eurofit ${ }^{6}$, pudiéndose realizar movimientos de los brazos antes del salto, pero sin permi- 


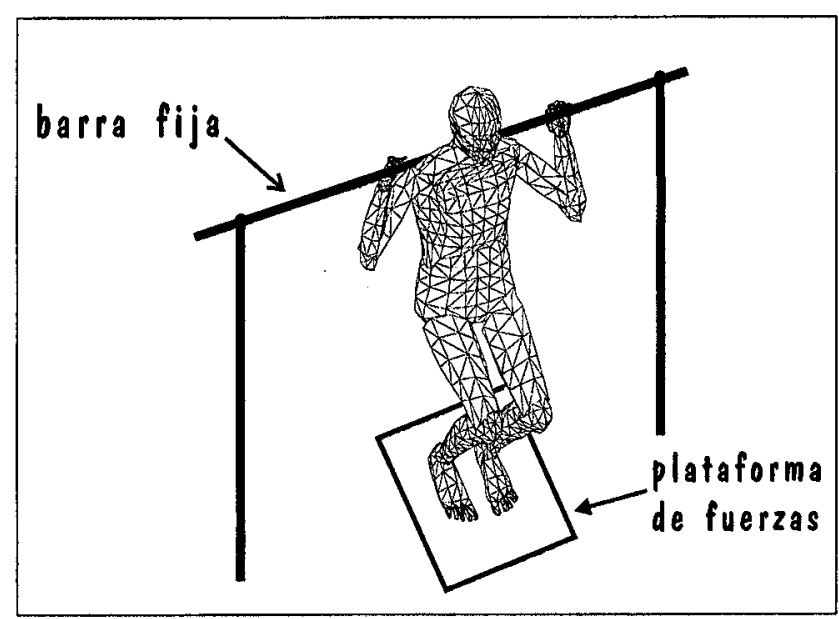

FIG. 1.-Esquema de la colocación del sujeto para la realización del test de fuerza isométrica máxima.

tir movimientos preparatorios de los pies antes de saltar. La distancia del salto se midió con una cinta métrica inextensible. Se realizaron tres saltos eligiendo el mayor para el análisis.

La fuerza explosiva isométrica, también se midió con una plataforma de fuerzas extensiométrica (Dinascan $600 \mathrm{M}, \mathrm{IBV}$ ). Se pidió a los sujetos que al oír una señal externa realizaran la máxima fuerza, tan rápido como fuera posible contra una resistencia inamovible. Así, se obtuvo la fuerza isométrica máxima bilateral (MFI) y la (PMF). Para ello, el sujeto estaba colocado de pie sobre la plataforma de fuerzas, con sus hombros en contacto con una barra fija, manteniendo ángulos de $90^{\circ}$ y $120^{\circ}$ de flexión de rodillas, que fueron las dos condiciones en que se realizó el test (Fig. 1). Se completaron tres intentos con descansos de $90 \mathrm{~s}$, escogiéndose el mejor para su análisis posterior. El análisis de la curva fuerzatiempo incluyó el cálculo de los impulsos de aceleración (IA) y frenado (IF) durante las batidas de los saltos $^{20,21}$.

Los registros de fuerzas pasaban desde la plataforma a una unidad electrónica externa y se introducían al ordenador (con un microprocesador Intel 486 Dx2 $66 \mathrm{Mhz}$ ) a través de una tarjeta de adquisición de datos.

\section{Muestras de fibra muscular y proceso de tinción}

A cada sujeto se le extrajo, en el vasto lateral del muslo derecho, mediante biopsia percutánea, una muestra cilíndrica de aproximadamente $4 \mathrm{~mm}$ de diámetro y $8 \mathrm{~mm}$ de longitud. Se realizó la extracción a $10 \mathrm{~cm}$ de la articulación de la rodilla ${ }^{22}$. Inmediatamente después de la extracción se montó la muestra en crioprotector (Tissue-Tek, O.C.T.) y se congeló en isopentano enfriado en nitrógeno líquido, almacenándose a $-80^{\circ} \mathrm{C}$ hasta el momento de hacer los cortes. Éstos se realizaron en un criostato a $-20^{\circ}$ con un espesor de $10 \mu$. Para la clasificación de las fibras musculares se practicó la reacción histoquímica de ATPasa a unos $\mathrm{pH}$ de preincubación de $10,3,4,6$ y 4,323-25. Finalmente, las fibras musculares se clasificaron en tipo I (slow-oxidative), tipo IIA (fast oxidative-glycolytic) y tipo IIB (fast glycolytic). Para estandarizar el procedimiento se contaron, por un solo investigador, una media de 250 fibras en cada sujeto.

El porcentaje de tejido graso fue estimado a partir de la medida de los pliegues cutáneos ${ }^{26}$.

\section{Análisis cinemático}

Se realizó un análisis cinemático $2 \mathrm{D}$ para determinar la velocidad de salida del centro de gravedad y la velocidad angular media de extensión de rodillas en el SHP, el CMJ y el SJ. Los tests de salto fueron grabados con una cámara de vídeo a $50 \mathrm{~Hz}$ (S-VHS Panasonic Ms1; Pal system). La cámara estaba colocada en una dirección perpendicular al plano de la batida. Después de las ejecuciones se colocó un sistema de referencia plano de dimensiones conocidas $(2.2 \mathrm{~m})$ en el mismo lugar donde se habían realizado los saltos. Los intentos seleccionados fueron digitalizados usando un digitizador sónico (Sac Graf/Bar Mark II) conectado a la salida Rs-232 del ordenador. Posteriormente se suavizaron los datos obtenidos mediante splines de la quinta potencia del método G.C.V. (Generalized Cross-Validation $)^{27,28}$ y se extrajeron las variables comentadas.

\section{Análisis estadístico}

Se utilizó el paquete estadístico Statistica for windows 4.5. Se usaron métodos estadísticos convencionales para el cálculo de las medias, desviaciones estándar (D.E.) y coeficientes de correlación de Pearson ( $r$ ). Se escogió el nivel de $p<0,05$ como el mínimo para establecer el criterio de significación estadística.

\section{Resultados}

Los valores medios ( \pm D.E.) de la distancia y altura de vuelo en el SHP, el SJ y el CMJ fueron respectivamente $2,50 \mathrm{~m} \pm 0,17,0,32 \mathrm{~m} \pm 0,05$ y $0,40 \mathrm{~m} \pm 0,05$. Los sujetos mostraron un similar porcentaje de fibras musculares de tipo I, entorno al $50 \%$. La distribución de las fibras musculares tipo I, IIA y IIB fue del $48 \% \pm$ $1,34 \% \pm 3$ y $17 \% \pm 5$, respectivamente.

En el SHP, se obtuvieron valores superiores en la velocidad de salida del centro de gravedad $(3,5 \mathrm{~m} / \mathrm{s} \pm$ $0,5)$ que en el SJ $(2,4 \mathrm{~m} / \mathrm{s} \pm 0,2)$ y en el CMJ $(2,3 \mathrm{~m} / \mathrm{s} \pm$ 
Tabla I. Media \pm D.E. de la altura y distancia, pico de máxima fuerza vertical, velocidad de salida del centro de gravedad, velocidad angular de extensión de rodillas, potencia mecánica y la relación entre el impulso de frenado (IF) y el impulso de aceleración (IA) en el squat jump (SJ), countermovement jump (CMJ) y salto horizontal a pies juntos (SHP)

\begin{tabular}{|c|c|c|c|c|c|c|c|}
\hline & $\begin{array}{l}\text { Desplaza- } \\
\text { miento (m) }\end{array}$ & $\begin{array}{c}\mathrm{FZ} \max / \mathrm{kg} \\
(\mathrm{N} / \mathrm{kg})\end{array}$ & $\begin{array}{l}V \max \\
(\mathrm{m} / \mathrm{s})\end{array}$ & $\begin{array}{c}\text { Vsalida } \\
\text { (m/s) }\end{array}$ & $\begin{array}{c}\text { Vext } \\
(\mathrm{rad} / \mathrm{s})\end{array}$ & $\mathrm{W} / \mathrm{kg}$ & IF/IA \\
\hline Squat jump .............. & $0,32 \pm 5$ & $2,6 \pm 0,5$ & $3,09 \pm 0,7$ & $2,4 \pm 0,2$ & $5,8 \pm 0,5$ & $56,5 \pm 1,1$ & - \\
\hline 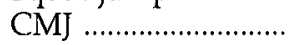 & $0,40 \pm 5$ & $2,8 \pm 0,5$ & $2,8 \pm 0,4$ & $2,3 \pm 0,5$ & $6,5 \pm 0,3$ & $64,4 \pm 4,2$ & $0,5 \pm 0,05$ \\
\hline 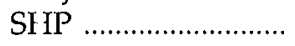 & $253 \pm 0,17$ & $2,3 \pm 0,5$ & $3,6 \pm 0,3$ & $3,5 \pm 0,5$ & $6,3 \pm 0,4$ & $63,4 \pm 5,4$ & $1,13 \pm 0,5$ \\
\hline
\end{tabular}

Tabla II. Valores expresados como media \pm D.E. para la fuerza máxima isométrica (MFI) y máxima pendiente de desarrollo de fuerza (PMF) durante acciones isométricas realizadas con un ángulo de flexión de rodillas de 120 y 90 grados

\begin{tabular}{|c|c|c|c|c|c|c|}
\hline & $\begin{array}{c}\text { MFI }\left(90^{\circ}\right) \\
(\mathrm{N})\end{array}$ & $\begin{array}{c}\mathrm{MFI} / \mathrm{kg}\left(90^{\circ}\right) \\
(\mathrm{N} / \mathrm{kg})\end{array}$ & $\begin{array}{l}\mathrm{PMF}\left(90^{\circ}\right) \\
(\mathrm{N} / \mathrm{s})\end{array}$ & $\begin{array}{c}\text { MFI }\left(120^{\circ}\right) \\
(\mathrm{N})\end{array}$ & $\begin{array}{c}\mathrm{MFI}\left(120^{\circ}\right) \\
(\mathrm{N} / \mathrm{kg})\end{array}$ & $\begin{array}{c}\text { PMF (120 }) \\
(N / s)\end{array}$ \\
\hline $\begin{array}{l}\text { Media } \\
\pm \text { D.E. }\end{array}$ & $\begin{array}{r}1538 \\
\pm 300\end{array}$ & $\begin{array}{r}2,06 \\
\pm 0,34\end{array}$ & $\begin{array}{l}7398 \\
\pm 1557\end{array}$ & $\begin{array}{l}2268 \\
\pm 576\end{array}$ & $\begin{array}{l}2,51 \\
\pm 0,5\end{array}$ & $\begin{array}{c}8962 \\
\pm 1945\end{array}$ \\
\hline
\end{tabular}

0,5) (tabla I). Sin embargo, en este último tipo de salto se obtuvieron valores inferiores en el pico de máxima fuerza vertical (1794 $\mathrm{N} \pm 554)$ en comparación con los obtenidos en el SJ y el CMJ (2072 N \pm 342 y $2228 \mathrm{~N} \pm 521$, respectivamente).

La velocidad angular de extensión de rodillas fue mayor en el CMJ $(6,5 \mathrm{rad} / \mathrm{s} \pm 0,3)$ que en el SHP $(6,3$ $\mathrm{rad} / \mathrm{s} \pm 0,4)$ y el SJ $(5,8 \mathrm{rad} / \mathrm{s} \pm 0,5)$ (tabla I). El pico de potencia mecánica resultó mayor en el SHP $(63,4$ $\mathrm{W} / \mathrm{kg} . \pm 5,4)$ que en el CMJ y el SJ $(64,4 \mathrm{~W} / \mathrm{kg} \pm 4,2$ y $56,5 \mathrm{~W} / \mathrm{kg} \pm 1,1$, respectivamente).

I a razón entre el IF y el IA ${ }^{20,21}$ en el SHP fue de 1,13 $\pm 0,65$, mientras que en el CMJ fue 0,5 $\pm 0,2$.

Tanto Ia MFI como la PMF fueron mayores $(\mathrm{p}<$ $0,001)$ cuando el test se realizaba con una flexión de rodillas de $120^{\circ}(2268 \mathrm{~N} \pm 576$ y $8962 \mathrm{~N} / \mathrm{s} \pm 1945$, respectivamente), que con $90^{\circ}(1538 \mathrm{~N} \pm 300$ y $7398 \mathrm{~N} / \mathrm{s}$ \pm 1557 , respectivamente) (tabla II).

La altura de vuelo conseguida en el SJ se correlacionó significativamente con la altura de vuelo conseguida en el CMJ ( $r=0,96 ; p<0,05)$ y con la distancia lograda en el SHP ( $r-0,97 ; p<0,05)$. Sin embargo, las correlaciones de las alturas y distancias de los saltos con la fuerza isométrica no fueron significativas (tabla III).

Tabla III. Coeficientes de correlación entre diferentes variables de tipo isométrico y dinámico

\begin{tabular}{|c|c|c|c|c|}
\hline & $\begin{array}{c}\text { MFI } \\
\left(120^{\circ}\right)\end{array}$ & $\begin{array}{l}\text { MFI } \\
\left(90^{\circ}\right)\end{array}$ & $\begin{array}{l}\text { PMF } \\
\left(120^{\circ}\right)\end{array}$ & $\begin{array}{r}\text { PMF } \\
\left(90^{\circ}\right)\end{array}$ \\
\hline 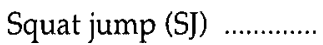 &,- 18 &,- 27 &,- 47 &,- 75 \\
\hline Count. jump (CMJ) ....... &,- 18 &,- 02 &,- 56 &,- 50 \\
\hline Salto horizontal (SHP) .. &, 08 &,- 40 &,- 28 &,- 78 \\
\hline
\end{tabular}

\section{Discusión}

Durante las acciones explosivas, los músculos deben desarrollar tanta fuerza como puedan en un corto período de tiempo. En los saltos verticales, la altura alcanzada estará determinada por la velocidad con la que despega el centro de gravedad y ésta a su vez,, con la fuerza que los músculos puedan generar durante el tiempo de la batida ${ }^{29}$ (Fig. 2).

En el $S H P$, se dispone de mayor tiempo en la batida, lo que favorecerá valores más altos en la velocidad de despegue del centro de gravedad. Sin embargo, la necesidad de contar en los saltos horizontales

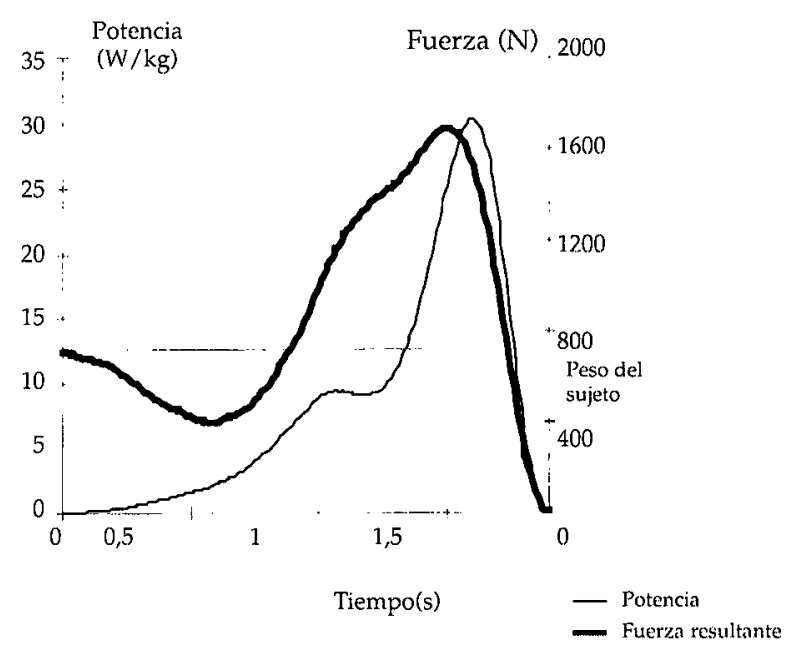

FIG. 2.-Curva de fuerza-tiempo y potencia al realizar un salto vertical con contramovimiento sobre las plataformas extensiométricas Dinascan $600 \mathrm{M}$ del Instituto Valenciano de Biomecánica - IBV- 


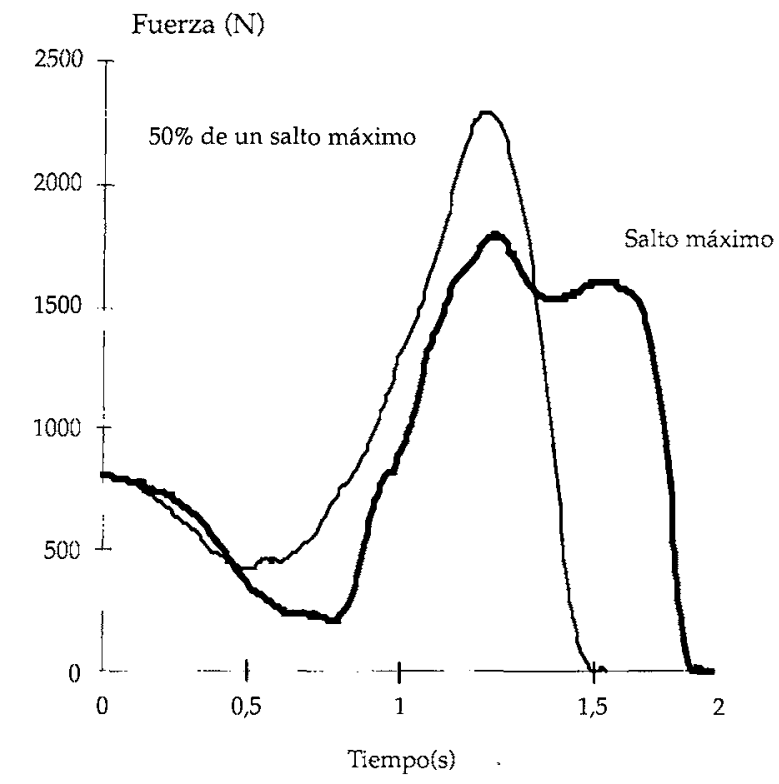

FIG. 3.-Representación gráfica de la fuerza vertical desarrollada por un mismo sujeto al realizar un test de salto horizontal a pies juntos máximo y otro a la mitad de su distancia máxima. El pico de fuerza vertical es mayor $(p<0,01)$ en el segundo acto.

con variables de eficacia no sólo relativas a la velocidad, sino también al ángulo de salida ${ }^{30}$, provoca que el valor de la fuerza vertical desarrollada pueda no ser un buen indicador de eficacia del salto. Como ejemplo, en la figura 3 se muestra cómo el pico de fuerza en un SHP máximo es inferior, en algunos sujetos, al que se obtiene cuando se realiza el mismo tipo de salto al 50\%. La explicación, desde el punto de vista biomecánico, es que en los saltos submáximos, obviamente, el sujeto más que en profundidad saltará en altura, originando a menudo un mayor valor en el pico de fuerza en comparación con el producido cuando el salto es máximo.

Por ello, desde el punto de vista de la fuerza explosiva serán poco válidos los parámetros de la PMF y la fuerza producida en los primeros $500 \mathrm{~ms}$ de la batida en el SHIP. Uno de los argumentos que apoyan esta idea, es el hecho de que la fuerza, por ejemplo, en un test de salto hacia adelante, no está directamente relacionada con el resultado de la acción (de tipo explosivo). Al contrario en el test isométrico, la acción y la fuerza producida estarán estrechamente vinculadas.

Desde el punto de vista de las características contráctiles de los diferentes tipos de fibras musculares, existe una considerable diferencia en la capacidad para desarrollar potencia entre las fibras tipo I y las fibras tipo II. Teóricamente, un sujeto con predominancia de fibras rápidas tendrá más capacidad para desarrollar potencia, ya que sus unidades motoras estarán mejor preparadas para producir una mayor tensión y tener una mayor frecuencia de estimulación ${ }^{31}$. Estos sujetos podrían llegar a alcanzar un pico de potencia hasta cuatro veces mayor en comparación con los que tengan predominancia de fibras tipo $\mathrm{I}^{32}$.

En el presente trabajo, a pesar de que los datos deben ser tratados con precaución debido al número limitado de sujetos, no se encontraron correlaciones significativas entre los valores de potencia en los diferentes saltos, los valores de fuerza isométrica máxima y los tipos de fibras musculares. De acuerdo con estudios previos, este conflicto se podría explicar en el caso de los saltos, por la capacidad para utilizar la energía elástica ${ }^{16,33}$, y en el caso de la producción de fuerza isométrica por el tamaño de las fibras musculares.

En un grupo de jóvenes ( $n=23)$ con una mayor variación interindividual en explosividad y distribución de fibras musculares, se observaron altas correlaciones entre características de la curva fuerza-tiempo isométrica y la producción de fuerza dinámica ${ }^{33}$. Las características musculares (similares en los diferentes sujetos de este estudio) explicarían en parte la ausencia de estas relaciones.

Autores como RHUTERFORD y JONES ${ }^{34}$ y ENO$\mathrm{KA}^{35}$, explican la precaución que hay que tener cuando se extrapolan resultados de los tests isométricos a acciones de tipo dinámico, ya que en estas últimas están influenciadas por factores como el aprendizaje y la capacidad de coordinación muscular.

Durante las contracciones isométricas, la forma de la curva f-t (Fig. 4), puede estar influenciada también por la cantidad y pendiente de activación neuronal ${ }^{14-36}$, el tipo de fibra muscular ${ }^{37}$ y el estado de entrenamiento del individuo ${ }^{38}$. En el presente trabajo, se observó cómo al realizar los tests isométricos máximos sobre la plataforma de fuerzas, se obtenían resultados diferentes, en función del ángulo de flexión de rodillas. Con un ángulo de $120^{\circ}$ todos los indivi-

Fuerza isométrica (N)

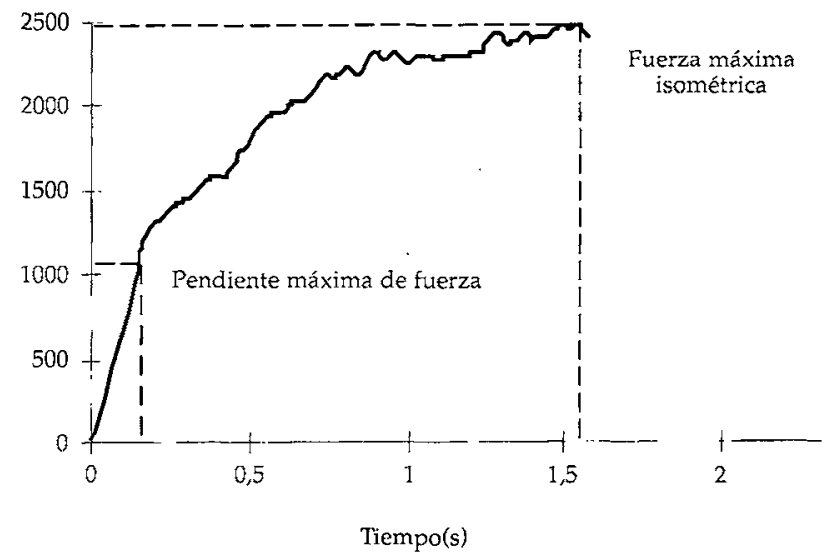

FIG. 4.-Curva fuerza-tiempo obtenida al desarrollar un test isométrico máximo sobre plataformas de fuerza. Se pueden ver la pendiente de máxima fuerza (PMF) y la máxima fuerza isométrica desarrollada (MFI) 
duos consiguieron sus mayores ejecuciones, tanto en la MFI como en la PMF. Estos resultados están en la misma línea de investigaciones previas ${ }^{39}$, en las que se muestra cómo la modificación del ángulo, puede cambiar las relaciones entre los tests isométricos y las acciones de tipo dinámico.

No se encontró relación significativa entre la producción dinámica de fuerza medida por medio de los tests de salto y las variables de fuerza isométrica. Entre las razones presentadas por los investigadores cuando explican el porqué de las escasas correlaciones entre los tests isométricos y dinámicos, se pueden destacar la influencia del patrón del movimiento ${ }^{40}$, la diferente activación neural ${ }^{41,42}$, la diferencia en el uso de la energía potencial de tipo elástico y la aceleración de los segmentos en las acciones de tipo dinámico, frente a la ausencia de éstas en las de tipo isométrico ${ }^{18,43}$. Esto explicaría la independencia entre algunas características de la curva $f-t$ isométrica y las acciones dinámicas ${ }^{33,44}$. También, la escasa correlación entre tests isométricos y dinámicos mostrada en distintos estudios ${ }^{45-49}$, lleva a pensar que ambas formas de investigar la fuerza explosiva podrían estar estimando cualidades musculares distintas $e$ independientes.

\section{Conclusiones}

En resumen, a pesar del tamaño reducido de la muestra, se confirmaron resultados de trabajos previos al no encontrar relaciones significativas entre las variables de fuerza isométrica y fuerza explosiva dinámica en los saltos. Sin embargo, se obtuvieron correlaciones estadísticamente significativas entre los distintos tests de salto.

En segundo lugar, se observó en los tests isométricos máximos la influencia del ángulo de flexión de rodillas sobre la pendiente y la máxima fuerza isométrica. Esto podría ser debido a diferentes patrones de activación nerviosa usados en las diferentes posiciones del movimiento, así como a los cambios en las propiedades mecánicas de mûsculo en cada posición. Además, la existencia de un ángulo optimo individual de flexión, no sólo podría influenciar la ejecución de los tests isométricos, sino también, la posición de partida en el salto vertical sin contramovimiento.

Las acciones musculares dinámicas no sólo parecen ser distintas a las isométricas desde el punto de vista fisiológico, sino también, al considerar la estructura del movimiento, el tipo y velocidad de contracción, así como, en la carga o resistencia a la que se le somete al individuo cuando realiza el test. Esto último sugiere que los test isométricos podrían no ser los más recomendables para analizar la capacidad funcional de la musculatura durante las acciones dinámicas.

Por último, señalar que el uso de las plataformas de fuerzas puede dar información valiosa no solo de as- pectos técnicos de la ejecución, sino también sobre parámetros de control y optimización de la fuerza explosiva.

\section{Bibliografía}

1. Bassey EI, Harries V. Force velocity characteristics of knee extensor muscles in young and elderly females. I Phys. 1987;384:32.

2. Häkkinen K. Neuromuscular adaptation during strength training, aging, detraining, and immobilization. Crit Rev Phys Rehabil Med. 1994;6:161-98.

3. Aguado X, Izquierdo M. La detente horizontal. Estudio cinemático y cinético de 64 casos en las pruebas de ingreso en el I.N.E.F. de León. Archivos de medicina del deporte, 1995.

4. Asmussen F, Bonde-Petersen F. Storage of elastic energy in skeletal muscles in man. Acta Physiol scand. 1974;91:385-92.

5. Bosco C, Komi P. Mechanical characteristics and fiber composition of human leg extensor muscles. Eur I Appl Physiol. 1979;41:275-84.

6. Council of Europe. Evaluation de l'aptitude physique. EUROFIT batterie expérimentale. Council of Europe. Strasbourg, 1983.

7. Aguado X. Eficacia en la técnica deportiva. INDE. Barcelona, 1993

8. Alway SE, MacDougall JD, Sale DG, Filder G, Sutton JR. UItrastructure of type I and type II fibers in human skeletal muscle. Med Sci Sports Exerc. 1981;13:95-6.

9. Coyle FF, Costill DL, Lesmes GR. Leg extension power and muscle fiber composition. Med Sci Sports. 1979;11:12-5.

10. MacDougall JD, Sale DG, Moroz. JR, Elder GCB, Sutton JR, Howald $\mathrm{H}$. Muscle ultrastructural characteristics of elite powerlifters and bodybuilders. Eur I Appl Physiol. 1982;48:117-26.

11. Komi PV. Physiological and biomechanical correlates of muscle function: effects of muscle structure and stretch-shortening cycle on force and speed. Exerc Sport Sci Rev. 1984;12:81-121.

12. Knuttgen $\mathrm{H}$, Kraemer WJ. Terminology and measurement in exercise performance. I Appl Sports Sci Res. 1987;1;1-10.

13. Thortensson A, Grimby G, Karlsson J. Force velocity relations and fiber composition in human knee extensor muscles. J Appl Physiol. 1976;40:12-6.

14. Viitasalo JT, Komi PV. Force-time characteristics and fibre composition in human leg extensor muscles. Eur J Appl Physiol. 1978;40:7-15.

15. Jaric S, Ristanovic D, Corcos DM. The relationship between muscle kinematic parameters and kinematic variables in a complex movement. Eur J Appl Physiol. 1989;59:370-6.

16. Viitasalo $I T$, Häkkinen $K$, Komi PV. Isometric and dynamic force production and muscle fibre composition in man. Scan I Sports Sci. 1981;2:199-208.

17. Mero A, Komi PV, Gregor RJ. Biomechanics of sprint running. Sports Medicine. 1992;13:376-92.

18. Wilson GJ, Lyttle AD, Ostrowski KJ, Murphy AJ. Assessing dynamic performance: a comparasion of rate of force development tests. I Strength Cond Res. 1995;9(3):176-81.

19. Young WB, Bilby GE. The effect of voluntary effort to influence speed of contraction on strength, muscular power and hypertrophy development. J Strength Cond Res. 1993;7:172-8.

20. Hochmuth G. Biomecánica de los movimientos deportivos. I.N.E.F. Madrid. Madrid, 1973.

21. Hochmuth $G$, Marhold $G$. The further development of biomechanical principles. En: Asmussen E \& Jorgenses K (Eds.): Biomechanics VI-B. Baltimore: University Park Press, 1977:93-106.

22. Bergström J. Muscle electrolytes in man. Scand J Clin Lab Invest. 1962; suppl. 68.

23. Brooke $\mathrm{MH}$, Kaiser KK. Three myosin adenosine triphosphatase systems: the nature of their pH lability and sulfhydryl dependence. J Histch Cytoch. 1970;18:670-2.

24. Komi PV, Karlsson J. Skeletal muscle fibre types, enzyme activities and physical performance in young males and females. Acta Physiol Scand. 1978;103:215-8. 
25. Costill DL, Fink WI. Analytical methods for the measurement of humans performance. Human Performance Laboratory, Ball State University, 1990.

26. Durnin J, Womersley $Y$. Body fat assessed from total body density and its estimation from skinfold thicknes: measurement on 481 men and women aged from 16 to 72 . Br J Nutr 1967;32:7797.

27. Clauser CE, McConville JT, Joung JW. Weight, volume and center of mass of segments of the human body. AMRL-TR-69-70. Aerospace Medical Research Laboratory, Wright-Patterson Air Force Base, Ohio, 1969.

28. Woltring HJ. On methodology in the study of human movement. En human motor actions. Elsevier Science Publisher. Amsterdam, 1984:35-7.

29. Newton RU, Kraemer JW. Developing explosive muscular power; implication for a mixed methods training strategy. Strength and conditioning. October, 1994:20-31.

30. Aguado X, Izquierdo M. 16 prácticas de Biomecánica. Universidad de León. León, 1995.

31. Sale D y cols. Determining factors of strength. Part I. NSCA. 1993:15:9-22

32. Faulkaner JA, Claflin DR, McCully KK. Power output of fast and slow fibers from human skeletal muscles. En: Jones y cols. Human muscle power. Champaing, IL Human kinentics, 1986.

33. Komi PV. Training of muscle strenght and power: interaction of neuromotic, hypertrophic and mechanical factors. Int J Sports Med. 1986;(suppl)7:10-5.

34. Rhuterford OM, Jones DA. The role of learning and coordination in strengtht training. Eur J Appl Physiol, 1979;55;100-5.

35. Enoka RM. Neuromechanical basis of kinesiology. 2nd ed Champaign (IL): Human kinetics, 1994.

36. Häkkinen $K$. Muscles crosswsectional area, force production and relaxation characteristics in males, females male and female strength athletes. En: Högfors $C$ (Ed.): Proceedings of the 3rd Biomecanics Seminar, Centre for Biomechanics, Chalmers University of Tecnology and Göteborg University. Göteborg, 1992:144-61.

37. Häkkinen $K$. Neuromuscular and hormonal adaptations during strength and power training. A review. J Sports Med. 1989;29:9-26.
38. Komi PV. Neuromuscular performance: factors influencing force and speed production. Scand J Sports Sci. 1979;1:2-15.

39. Murphy JA, Wilson JG, Pryor FJ. Use of the isoinertial force mass relationship in the prediction of dynamic human performance. Eur J Appl Physiol. 1994;69:250-7.

40. Baker D, Wilson $G$, Carlyon B. Generality versus specificity: a comparasion of dynamic and isometric measures of strength and speed-strength. Eur J Appl Physiol. 1994;68:350-5.

41. Nakazawa K, Kawakami $Y$, Fukunaga $T$ y cols. Differences in activation patterns in elbow flexors during isometric, concentric and eccentric contractions. Eur J Appl Physiol. 1993;66;21420 .

42. Caldwell G, Jamison J, Lee S. Amplitude and frequency measures of surface electromyography during dual task elbow torque production. Eur J Appl Physiol, 1993;66:349-56.

43. Abernethy P, Wilson G, Logan P. Strength and power assessement: issues, controversies and challenges. Sports Med. 1995;6: 401-13,

44. Kao VT. Factors conditioning jumping heigth of volleyballs players. Teoriya i Praktika Fizicheskoi Kultury. 1971;4:18-20.

45. Fry AC, Kraemer WJ, Weseman CA y cols. Effects of an off-season strength and conditioning program on starters and nonstarters in women's collegiate voleyball. J Appl Sport Sci Res. 1991;5:174-81.

46. Anderson MA, Gieck JH, Perrin D y cols. The relationship among isometric, isotonic and isokinetic concentric and eccentric cuadriceps and hamstring force and three components of athletic performance. J Orthop Sports Phys Ther. 1991;14(3): 114-20.

47. Komi PV, Suominen H, Heikinnen E y cols. En: Komi PV (Ed.): Exercise and sports biology. Champaign, IL: Human Kinetics. 1989:90-102.

48. Viitasalo JT, Era P, Leskinen AL, Heikkinen E. Muscular strength profiles and anthropometry in random samples of men aged 31 to 35,51 to 55 and 71 to 75 years. Ergonomics. 1985a;28:1503-74.

49. Viitasalo JT. Measurement of force-velocity characteristics for sportsmen in field conditions. En: Winter DA, Norman RW, Wells RP y cols. (Eds.): Biomechanics IX-A. Champaign. IL: Human Kinetics. 1985b:96-101. 